\title{
Specifications for signal processing and data handling used for infant pulmonary function testing
}

\author{
U. Frey*, J. Stocks**, P. Sly***, J. Bates ${ }^{+}$, on behalf of the ERS/ATS Task Force on Standards for \\ Infant Respiratory Function Testing
}

Specifications for signal processing and data handling used for infant pulmonary function testing. U. Frey, J. Stocks, P. Sly, J. Bates, on behalf of the ERS/ATS Task Force on Standards for Infant Respiratory Function Testing. (C) ERS Journals Ltd 2000.

ABSTRACT: The aim of this present paper is to define minimal performance criteria for the separate items comprising signal processing and data handling used to measure respiratory function in infants. These guidelines cover numerous aspects including: signal processing, data handling and subsequent analysis, reporting of results, demographics and handling of reference values. Adherence to these guidelines should ensure that infant lung function measurements can be performed with an acceptable degree of safety, precision, and reproducibility. Furthermore, they will facilitate multicentre collection of data and the performance of clinical investigations. Eur Respir J 2000; 16: 1016-1022.

*Dept Paediatrics, University Hospital of Bern, Bern, Switzerland. **Portex Anaesthesia, Intensive Therapy and Respiratory Medicine Unit, Institute of Child Health, London, UK. ***Division of Clinical Sciences, University of Western Australia, West Perth, Western Australia, Australia. ${ }^{+}$Vermont Lung Centre, The University of Vermont, Colchester, Vermont, USA

Correspondence: U. Frey, Dept Paediatrics, University Hospital of Bern, 3010 Bern, Switzerland. Fax: 41316329484

Keywords: Equipment, infants, lung function, signal processing, software, standardization

Received: March 282000

Accepted after revision June 142000

This work was supported by a grant from the European Respiratory Society, and by donations from Glaxo-Wellcome(UK) and Glaxo-Wellcome AB (Sweden).
The present document represents the third of a series [1$4,6,7]$ that is being produced by the European Respiratory Society/American Thoracic Society Task Force on standards for Infant Respiratory Function Testing. The aim of this task force is to summarize what is currently seen to be good laboratory practice, and to provide recommendations for both users and manufacturers of infant lung function equipment and software. These recommendations have been developed after widespread communication on an international level and are directed towards future developments in this field, including the use of more automated equipment than has been used in many research centres in the past.

As the technology for assessing respiratory function expands and progresses, it will become increasingly necessary to have the ability to compare results between systems in a coherent fashion. The feasibility of performing multicentre trials to investigate infant respiratory physiology or study the effects of disease and therapeutic interventions on the developing lung, has been limited to date by the wide range of equipment and software that has been used. The lack of standardized equipment and software has also made it difficult to establish normative values for the various parameters of interest that are independent of the measurement device used, or to use these tests as reliable clinical tools.

In a previous study [1] the minimal hardware criteria for equipment used in infant lung function testing and details of data using an analog-to-digital (A/D) converter have been specified. However, once signals have been acquired they need to be processed in order to take account of prevailing measurement conditions, and correct for any signal drift. When analysing respiratory signals in infants and young children, particular attention is required with respect to factors such as accurate breath detection, and determination of a representative end

Previous articles in this series: No. 1: U. Frey, J. Stocks, A. Coates, P.D. Sly, J. Bates, on behalf of the ERS/ATS Task Force on Standards for Infant Respiratory Function Testing. Specifications for equipment used for infants pulmonary function testing. Eur Respir J 2000; 16: 729-738. No. 2: P.D. Sly, R. Tepper, M. Henschen, M. Gappa, J. Stocks, on behalf of the ERS/ATS Task Force on Standards for Infant Respiratory Function Testing. Tidal Forced Expirations.Eur Respir J 2000; 16: 739-746. 
expiratory level. Minor differences in algorithms may lead to major differences in reported results, especially when studying small, rapidly breathing infants. Therefore, it is essential that manufacturers provide the user with details of all algorithms used for calculations, including insight into those used for applying corrections such as linearization, drift correction and so forth, and the means to verify the validity of such algorithms.

The aim of the present paper is to describe the general principles of signal processing and data handling for infant respiratory function tests, and then provide guidelines regarding the display, analysis, reporting and storage of such data.

\section{Signal processing}

Raw signals from the A/D board are usually processed and displayed after undergoing some corrections and modifications. As stressed earlier, these signal processing algorithms should be described in the operator's manual.

\section{Drift correction}

Signal drifts occur due to baseline instability of the pressure or flow transducers, changes in temperature, gas composition or ambient pressure, or the limitations of the signal processing algorithm (e.g. during flow integration) $[1,2]$. If there is a constant upward or downward drift in signals due to such factors, this trend should be removed to force the signal to vary from breath to breath about a stable baseline. Different drift removal algorithms will usually lead to slight differences in the values of breath parameters subsequently estimated and the optimal approach will depend on the specific parameter in question. However, given that drift correction is merely an empirical operation, it is probably appropriate to select a correction algorithm on the basis of robustness and ease of implementation as much as anything else. Tidal volume [2], plethysmographic pressure [3], and signals such as oesophageal pressure derived from microtip catheters are the signals most likely to need drift correction.

For accurate infant lung function measurements some means of reliably rezeroing flow during data collection as well as analysis is essential. Any constant drift of the end expiratory level, e.g. due to slight flow off-set, or changes in inspired/expired gas composition and characteristics, must be corrected prior to data analysis [2]. However, it must be recognized that under certain measurement conditions a true shift in functional residual capacity (FRC) may occur. Under such circumstances application of a drift correction would be inappropriate. Drifts can be corrected by hardware (voltage offset, high pass filters) or software rezeroing algorithms. If high pass filters (e.g. $>0.1 \mathrm{~Hz})$ are used, filter characteristics should not influence the amplitude or phase of the measured signal over a specified range. This can be achieved approximately by using analog filters. Drift correction is discussed in more detail elsewhere [2].
Correction for body temperature, ambient pressure, and saturation with water vapours

Corrections for temperature changes, ambient pressure, and saturation with water vapours (BTPS) at the airway opening are difficult to predict. Inspired/expired differences are significantly influenced by the environment of the inspired air (e.g. heated incubator). Exhaled gas flow temperature measurements made at the mouth appear to be between $30-35^{\circ} \mathrm{C}$. BTPS correction can be made if all temperature and humidity values are known. Particular problems occur if respired air differs from ambient room conditions. This is the case if cooler, dry air or oxygen supplied from cylinders or wall supplies are used, for example during raised volume manoeuvres, or warm, humidified air is used during plethysmographic airway resistance measurements. This is one of the most difficult areas in which to define a standard. The major unknowns affecting the correction are the temperature and humidity at the flow sensor. Since it is not feasible to undertake the necessary conditioning of inspired gas, the application of an appropriate correction factor is recommended. Currently some infant pulmonary function test (PFT) systems make no adjustment, others correct inspired gas using the equation:

$$
\begin{aligned}
V^{\prime} \text { BTPS }= & V^{\prime} \text { ATP } \times 310.2\left(P \mathrm{amb}-P \mathrm{H}_{2} \mathrm{O}\right) /((273.2+t) \\
& \times(P \mathrm{amb}-6.3))
\end{aligned}
$$

where $V^{\prime}$ ATP is the flow at ambient temperature, $\mathrm{t}$ is the ambient temperature $(\mathrm{C}), \mathrm{Pamb}$ is the ambient barometric pressure $(\mathrm{kPa})$, and $\mathrm{PH}_{2} \mathrm{O}$ is the water vapour pressure $(\mathrm{kPa})$ of the ambient gas.

Other systems apply a partial correction to both inspired and expired flows, to take into account the potential deconditioning of expired gas by the upper airways before it passes through the pneumotachograph (PNT). This leads to systematic differences in recorded flows and volumes between systems. While acknowledging the limitations, it is suggested that, until further evidence is available, inspiratory flows and lung volumes by gas dilution should be corrected to BTPS using Equation 1 in order to minimize systematic differences between systems. Ideally, the user needs to be able to enter current temperature and humidity details not only from day-to-day/subject-tosubject according to ambient conditions, but with respect to the prevailing measurement conditions during any particular test (e.g. tidal breathing versus raised volume techniques).

It should be remembered that BTPS correction is not always required, for example during plethysmographically determined lung volumes, or flow and volume changes during either airway resistance measurements using a heated humidified system [3], or forced expired manoeuvres [4].

\section{Breath detection}

It is essential that the measuring device can accurately determine the point of zero flow for identification of the respiration phase. Particular care is required when studying small infants with low mean flows in whom inappropriate use of a flow "dead band" may lead to marked errors in detecting the start of inspiration and expiration [2]. Any drift correction should be applied prior to breath 
recognition algorithms. The breath detection algorithms are discussed in more detail elsewhere [2].

\section{Identification of end expiratory level}

It is vital to establish a representative baseline end expiratory level (EEL) for virtually all infant respiratory function tests (especially plethysmography, gas dilution methods, multiple occlusion technique and the rapid thoraco-abdominal compression technique). This should be calculated at the same time as the application of any necessary drift correction to tidal volume [2]. The calculated EEL must be displayed clearly on the time-based trace so that the user can evaluate whether a representative level has been selected. Excessive drift might alter the accuracy of signal processing. A warning should appear if drift correction is too large (e.g. slope $>10 \%$ of the signal in question). This will depend on the signal of interest, but for tidal volume a drift correction of $>10 \%$ would almost certainly indicate either a leak requiring repositioning of the mask, or a marked flow offset requiring recalibration of the $\mathrm{PNT}$.

The detection of EEL is discussed in more detail elsewhere [2].

\section{Viscosity correction}

Pneumotachographs are sensitive to gas viscosity. For example, the error introduced in PNTs and some anemometers by the differences in viscosity between room air calibration and measurements in $100 \%$ oxygen is $11-12 \%$. Although correction of the PNT signal for gas viscosity may be achieved by calibration with gases of the same composition as during the test, this requires considerable skill and is time consuming. It is therefore, necessary to mathematically correct for differences in gas composition [5]. Manufacturers should supply details of the algorithms used for such corrections. The most relevant gases that need to be considered are oxygen, carbon dioxide, nitrogen, nitrous oxide, helium and sulphur hexafluoride. Anemometers which are sensitive to viscosity should be corrected in a similar fashion. Ultrasonic flow-meters measure flow independently of gas viscosity.

\section{Barometric pressure correction}

Anemometers are sensitive to changes in barometric pressure and should be corrected for those changes. This is a physical characteristic as reported by the manufacturers of these devices.

\section{Display of data during recording and analysis}

This is essential both for safety reasons and to facilitate and speed up data collection and also to provide essential quality control during analysis. Display options should be available as follows: 1) As a safety measure a continuous display of at least tidal volume or flow (and ideally also mouth pressure to detect any sudden increase in apparatus resistance) is essential at all times when the mask and apparatus are connected to the infant (i.e. not just during data collection). 2) The user should be given the opportunity to inspect recorded data, and exclude any that is technically unsatisfactory, prior to saving. 3) During measurements simultaneous time-based displays of appropriate channels and X-Y plots (e.g. flow-volume loops) are needed. These should be of sufficient size and resolution to assist quality control during data collection, analysis and validation procedures. 5) The channels displayed for any given measurement technique should be set as defaults but be adaptable by the user if necessary. 6) Sensible defaults for scaling of the pressure, flow and volume axes should be set according to the specific test and infant weight (e.g. a volume axis based on tidal volume $\mathrm{kg}^{-1}$ ). Automatic rescaling may be advantageous for rapid lung function measurements in infants, however, there should be an option to disable automatic scaling and adjust manually if required. Where appropriate, cumulative plots of manoeuvres should be displayed. This is important for quality control, assessment of reproducibility and trend analysis of the data. 7) The software should provide the option to overlay data when required, e.g. tidal and forced flow volume curves. 8) Whenever possible preliminary analysis of results should be performed on-line during data collection. However, subsequent off-line examination and amendment of the results is essential for quality control.

\section{Data handling and analysis}

Guidelines regarding appropriate algorithms for data analysis of the various lung function techniques are discussed in the test specific documents in this series [2-4, $6,7]$. However, some general issues are summarized.

\section{Mathematical computations and references}

The equations used to calculate any nonprime variable (i.e. those not directly measured from a single signal including time) should be documented and available to the operator. Similar documentation is required for the equations used to correct any of the prime variables (e.g. drift, gas composition or BTPS correction), together with a list of references to support the use of specific equations.

\section{Data handling}

In order to facilitate selection, storage, analysis and data transfer, infant lung function software platforms should provide the following features: 1) The user should be able to readily import and export data into and from the program as American Standard Code for Information Interchange format (ASCII) and/or other frequently used formats (Symbolic link format (SYLK), binary format (BIN), data interchange format (DIF), etc.). 2) The user should be able to display data as time based and X-Y plots and reanalyse it blind to previous investigations. 3) For many tests the final evaluation can only be performed offline by interactive operator control (e.g. selection of suitable intervals for tidal breathing analysis [2]; determination of the pressure plateau during an occlusion or the 
most appropriate portion of the flow volume curve for regression analysis, during the single breath technique [7]). This must be supported by appropriate software. 4) The user should be able to view data and accept/reject specific manoeuvres both pre- and post analysis. During data collection the option to disregard technically unsatisfactory manoeuvres should be provided. However, during subsequent off-line analysis it should be possible to exclude but not delete, apparently unsatisfactory data from the statistical summaries. 5) Error messages should warn of any system failures or if technically unacceptable data (e.g. Ieak) are detected. The latter depends on close cooperation between the manufacturers and end users to avoid un-

necessary warnings. 6) Analysis options (e.g. number of breaths to analyse, duration of occlusion etc.) should be set to appropriate default values according to the recommendations of the Infant Lung Function Task Force, taking into account the size and clinical condition of the infant being studied [2-4, 6, 7]. However, since no such algorithms are likely to work all of the time, the analysis options should be easily accessible and user-definable. 7) The user must be able to check that various criteria for technically satisfactory measurements have been achieved e.g. by suitable graphic or tabular display of appropriate signals. 8) The user must be able to add comments when collecting and analysing data. 9) All relevant information (including biometric data, analysis options, calibrations, filters, sampling rate etc.) should be stored to facilitate subsequent examination and reanalyses. 10) Relevant statistical summaries of the results must be provided, together with the facility to recalculate the summary if data are subsequently excluded. These summaries will vary according to the parameter of interest. For research purposes the use of mean \pm standard deviation (SD) is usually preferred, whereas in a clinical setting the use of medians ( \pm range) may be more robust. A major limitation of the latter is the inability to calculate weighted means when data are collected during several different trials and the difficulties of subsequent statistical analysis of group data. 11) The calculation of a true weighted mean if data are collected during more than one epoch is required. 12) The ability to readily export results (all trials or summary statistics for selected parameters) in standard spreadsheet, database or any other specified file format (ASCII, BIN etc.) is essential.

\section{Storage of data}

The amount of data which needs to be stored will depend partly on whether collaborative multicentre studies are being performed, new analytical methods developed, or the study is primarily for clinical information on an individual patient basis. However, any software developed should always save the patient information plus calibration checks, essential details of signal processing (sampling frequency, filtering, thresholds etc.) and provide the user with the option to save the following: 1) Appropriate calibrated raw data, so that the original test can be displayed and reanalysed if desired. The operator needs to verify which data should be saved for each test to avoid storing unwanted data sets. 2) Details of prevailing measurement conditions including the total duration of the test and any periods when recordings ceased (because infant woke, needed feeding etc.). Order of testing can potentially influence results, as can the order of and duration between manoeuvres within a given test (changes in sleep state etc.). Consequently the actual time of data collection must be clearly displayed on all data sets and subsequent analyses. 3) Summary of total number of trials performed. 4) Results of individual trials. 5) Summary of accepted results together with specified graphical display (e.g. flow volume curves). 6) Technically satisfactory data that prove to be outliers should be saved, even if not reported. 7) Comments and notes regarding the individual measurements.

\section{Demographics}

While not the responsibility of manufacturers, most measurements of infant respiratory function are highly dependent on the weight, height, gender and age of the child. It is therefore, essential that these parameters are measured and documented accurately [5]: 1) Weight should be recorded in $\mathrm{kg}$ to 3 significant digits. 2) Crown heel length at test should be measured by two individuals, including at least one trained operator using a calibrated stadiometer (not a tape measure). Measurements should be repeated until two are obtained within $0.5 \mathrm{~cm}$, and the result recorded in $\mathrm{cm}$ to 1 decimal place. 3) Regular (weekly) calibration of scales and stadiometer using standard weights and rods and 3-6 monthly manufacturer service is recommended.

To assist in interpreting, comparing or collating data within and between centres, certain essential information should be recorded at the time of each test in a standardized fashion. It must be possible for the contents of such a database to be exported in standard spreadsheet, database or ASCII format. Suitable platforms should therefore, be provided to allow the user to enter and subsequently readily retrieve the following information.

Background information. Background information may vary depending on the test, age or patient group studied. Therefore, only general suggestions regarding background information can be made. However, subsets of this information might be selected for a particular test procedure. The authors suggest: date of birth (DOB); date of test (DOT); time and duration of test; birth weight and gestational age; ethnic group and sex; addresses, telephone number and names of parents, (plus grandparents, if it is a longitudinal study); family doctor, paediatrician (if appropriate); insurance (if relevant); identity numbers including laboratory/hospital identifier, hospital number (infant+mother), project number (if appropriate), and any other clearly defined identity number.

An important point to note is that since confidentiality and the Data Protection Act must be observed at all times some of this information may need to be stored separately.

Infant details at time of test. The details should include: age (calculated from DOB and DOT and displayed in sensible units, i.e. days if $<1$ month (to 2 decimal places (DP) when necessary, to allow for those $<24 \mathrm{~h}$ ), weeks or months to 1 DP for infants 1-24 months of age, decimal years, to 1 DP thereafter). Conversion algorithms should be available to 
the user to convert between these formats, and data should be saved internally in an appropriate form to allow export or reporting in decimal weeks, months or years as desired; test weight and length; diagnosis and/or reason for undertaking test; current symptoms, medication; sedation details.

Measurement conditions at time of test. These should include: type and size of face mask and sensors used (e.g. PNT, oesophageal pressure catheter, capnograph): apparatus deadspace, resistance etc. (this may differ throughout the study according to tests applied); barometric pressure, temperature, humidity, and altitude; calibration checks.

It is important to remember that some if not all of these details will need to be stored with the actual test results.

\section{Other potentially useful information to record}

Depending on the type of study, other relevant information will need to be documented. This could include factors such as: pre and postnatal exposure to tobacco smoke; family history of respiratory problems, including asthma in first degree relatives; obstetric complications or complications at delivery; neonatal medical history if relevant, and birth centile; current medication, respiratory support (e.g. inspiratory oxygen fraction, continuous positive airway pressure, positive inspiratory pressure (PIP)); maternal age, height and booking weight; socioeconomic factors.

\section{Reference values}

Inappropriate use of reference values [5, 8-12] can lead to more errors due to misinterpretation of the results in both clinical and research studies than inadequate equipment. Based on the issues discussed in the Appendix, some recommendations regarding the use and display of reference values can be made as follows: 1) Manufacturers and users are urged to exercise caution when expressing results in relation to previously published "reference data", especially when these have been collected using different equipment and software or in different laboratories. 2) Manufacturers should always quote the origin of the reference ranges used. 3) Manufacturers should not use reference values from cross-sectional data to interpret longitudinal data sets in individual infants.

Reference values from the local population may not be in accordance with published normative values particularly if there are ethnic or social differences. In such cases establishing local reference values or recruiting an appropriate control group is recommended. Percent predictions' have the disadvantage of not providing any information about the normal biological intersubject variability in healthy infants. For clinical assessment, the use of reference ranges (e.g. defined by cut-off values, SD $(Z)$ scores or percentiles) is therefore, more appropriate.

\section{Back-up policy}

The use of tape streamers, jaz drives, writeable compact discs or zip drives to ensure fail-safe and efficient back-up of the very large amounts of data that can be generated during research studies and to safeguard precious clinical and research databases is recommended. Recommendation and provision of suitable devices by the manufacturers would be helpful. Each laboratory should also ensure that they have a suitable back-up policy that is adhered to rigorously. Information on patients' diagnosis or personal information must be password protected. Special care has to be taken if infant lung function equipment is implemented into computer networks.

\section{Design of reports}

Design of reports will vary according to the array of tests applied and should be flexible enough to allow individual tailoring to the needs of different laboratories/clinics. Reports should allow the import of relevant background information, previously tabulated results plus some interpretation with respect to normal ranges section, graphics (e.g. representative flow-volume loops) and space for free text. The ability to print out all signals and loops with sufficient resolution and quality for assessment and further evaluation is extremely helpful when validating new programs or a particularly difficult set of data. The facility for trend reports with appropriate time axes should be available, so that all previous measurements in an individual child can be collated. However, this will be difficult to achieve for infants until reliable "growth curves" and Z-scores for lung function have been established over this age range. Respiratory function results should focus on the most relevant outcomes to prevent information overload. Results may be expressed as mean \pm SD, median+range, Z-score or individual values depending on the type of data.

Details concerning the relevant outcome measures, normative values and the optimal statistical way of expressing results are provided in the more specialized papers $[2-4,6,7]$. However, software must include the facility to specify and enter appropriate reference equations and the option to express results in relation to some agreed standards, as well as locally available control data. Such reference equations, once established, need to be documented in the operator's manual, and be appropriately referenced.

\section{Standard abbreviations, definitions and units}

The use of standard abbreviations, definitions and units for all the parameters of infant PFT, as recommended by the ERS and ATS $[12,13]$ is strongly suggested. However, it is recognized that some adaptation will be necessary due to the limiting factors of a majority of the software with respect to length of variable name and the inability to cope with certain characters such as primes. Results can be displayed and stored using either short (68 digits) or long ( 25 digits) entries providing a clear list of definitions is provided for the user. Further details are provided in the accompanying documents dealing with specific techniques. [2-4, 6, 7].

\section{Conclusions}

In the present paper recommendations have been provided regarding the minimum technical requirements for infant PFT signal processing and data handling, so that 
the measurements can be performed with an acceptable degree of precision, and reproducibility. In terms of signal processing, drift, viscosity, barometric pressure BTPScorrections, identification of EEL and breath detection algorithms have been discussed. For safety reasons and to facilitate data collection, recommendations have also been given regarding the display of data during recording and analysis. In order to facilitate the selection, storage, analysis and data transfer of infants lung function data, minimal criteria for: data handling, storage, mathematical computations, reference values, demographics, clinical and biometric background information, has been summarized. Particular emphasis has been placed on the importance of documenting and storing technical details of the apparatus, measurement conditions and calibration checks. Finally, some recommendations on how the test results should be reported have been provided.

The purpose of this paper is to facilitate quality control through adequate documentation and assessment of both equipment and algorithms. This will reassure the end user that the system can be used with a degree of confidence and that results will be compatible with those attained with other systems. It is recognized that this document will need regular updating in response to advances in both technology and the knowledge regarding the application and interpretation of these tests under different circumstances. In the meantime, every attempt has been made to avoid being too prescriptive to allow for future developments, while at same time offering guidance as to the minimum standards for those developing equipment and performing tests. These standards will facilitate international collaboration, the exchange of clinical pulmonary function testing data for second opinions, and training.

\section{Appendix: reference values}

The use of predicted values based on reference ranges has many intrinsic problems and pitfalls. The positive predictive value is the probability that a subject with a positive test really has the disease or disorder. Conversely, a negative predictive value is the probability that a subject with a negative test result is truly free of the disease or disorder. These measures are generally of more importance to the subject being tested and the clinician than either sensitivity or specificity. Unlike sensitivity or specificity, the predictive value depends on the prevalence of the disease or disorder in the group being tested. Tests that initially seem very promising may often be disappointing when applied to populations with a lower than expected prevalence of the outcome of interest. Thus, the use of reference values from sources other than local healthy populations, or pooled data sets with differences in prevalence of the examined disorder, may be misleading.

The use of reference ranges is similarly difficult. The reference range gives the probability that an individual infant's value is different from the majority of values observed in the population of similar infants who were used to derive that reference range. An underlying question is whether an improbable value indicates that the individual is in some way abnormal or at higher risk of an adverse outcome. The definition of what an "abnormal value" is depends on the sample size and distribution of the reference values, the repeatability of the parameter in question, in addition to how well the test relates to the outcome in which the investigator is interested. The latter, once again, might relate to epidemiological aspects of the disorder in the local population. All these factors may contribute to the misinterpretation of measured values in relation to published reference ranges $[10,11]$.

Both manufacturers and users need to be aware that no generally valid reference values for any infant lung function test are currently available. While many research groups have reported so called "normative data", this is generally only applicable to their specific population (bearing in mind ethnic, social and age related factors), their specific equipment and the type of respiratory function test used (i.e. values of resistance will vary markedly according to the measurement technique used). This is one of the main reasons why lung function tests have not been widely used to date, as a clinical tool in infancy.

The criteria used to select a reference population should be clearly specified [5]. The GAP conference [8] described criteria that may be used to define a population suitable for the development of reference ranges. For the "ideal" reference group this includes: no present acute or past or present chronic condition of the respiratory tract; no major respiratory disease; no major systemic disease which directly or indirectly influences the respiratory tract; no more than incidental antenatal or postnatal smoking exposure; no recent upper tract infection (in the past 3 weeks); appropriate growth pattern for gestational and postnatal age.

However, this "ideal" reference group might not correspond to the "representative" of a given population, in which for example prevalence of pre- and postnatal smoke exposure might be very high. Thus, the reference group used must be appropriate for the question posed during either clinical or research studies. Problems may also arise when interpreting serial or longitudinal studies in individuals or groups of individuals, most reference data in this field are based on single rather than serial measurements within individuals. Using cross-sectional data to interpret longitudinal data may be misleading, as the centiles derived from cross-sectional data are not a reflection of how an individual will change with time.

The way to solve some of these problems in the future may be to pool data between centres that have employed similar techniques, equipment and software. However, it must be kept in mind that differences between centres with respect to populations and precise methodology may still limit the potential value of this approach unless considerable effort is made to take such factors into account.

Acknowledgements. The authors would like to thank all other members of the Task Force who contributed to developing these recommendations, namely: J. Allan (Philadelphia, USA), E.B. Yishay (Jerusalem, Israel), C. Beardsmore (Leicester, UK), R. Castile (Colombus, USA), J.B. Clough (Southampton, UK), A.L. Coates (Toronto, Canada), I. Dundas (London, UK), D. Filbrun (Colombus, USA), M. Gappa (Hannover, Germany), S. Godfrey (Jerusalem, Israel), I. Goetz (London, UK), P. Gustafsson (Skövde, Sweden), R. Gregson (Southampton, UK), M. Henschen (Freiburg, Germany), A-F. Hoo (London, UK), A. Jackson (Boston, USA), J. de Jongste (Rotterdam, the Netherlands), R. Kraemer (Bern, Switzerland), S. Lum (London, UK), 
P. Merkus (Rotterdam, the Netherlands), I.T. Merth (Almelo, the Netherlands), M. Morris. (Little Rock, USA), B. Reinmann (Bern, Switzerland), G. Schmalisch (Berlin, Germany), P. Seddon (Brighton, UK), G. Sharma (Chicago, USA), M. Silverman (Leicester, UK), R. Tepper, (Indianapolis, USA), D. Vilozni (Petach-Tikva, Israel), E. van der Wiel (Rotterdam, the Netherlands), and to members of the industry who provided invaluable feedback.

\section{References}

1. Frey U, Stocks J, Coates A, Sly P, Bates J. Standards for infant respiratory function testing: Specifications for equipment used for infant pulmonary function testing. Eur Respir J 2000; (in press).

2. Bates J, Schmalisch G, Filbrun D, Stocks J. Standards for infant respiratory function testing: Tidal breath analysis for infant pulmonary function testing. Eur Respir $J 2000$.

3. Sly P, Tepper R, Henschen M, Gappa M, Stocks J. Standards for infant respiratory function testing: Tidal forced expirations. Eur Respir J 2000.

4. Gappa M, Sly P, Stocks J. Standards for infant respiratory function testing: Passive respiratory mechanics: The occlusion techniques. Eur Respir J 2000.

5. Stocks J, Sly PD, Tepper RS, Morgan WJ. Infant Respiratory Function Testing. Wiley-Liss, New York 1996.

6. Stocks J, Godfrey S, Beardsmore C, Bar-Yishay E, Castile R. Standards for infant respiratory function testing: Plethysmographic measurements of lung volume and airway resistance. Eur Respir J 2000.

7. Morris MG, Gustafsson P, Tepper R, Gappa M, Stocks J. Standards for infant respiratory function testing: The bias flow nitrogen washout technique for measuring functional residual capacity. Eur Respir $J 2000$.

8. Taussig LM, Chernick V, Wood R, Farrell P, Mellins RB. Standardisation of lung function testing in children. Proceedings and recommendations of the GAP conference committee, Cystic Fibrosis Foundation. J Pediatr 1980; 97: 668-676.

9. Quanjer PH, Stocks J, Polgar G, Wise M, Karlberg J, Borsboom G. Compilation of reference values for lung function measurements in children. Eur Respir J 1989; 2 (Suppl. 4): 184s-261s.

10. American Thoracic Society. Lung function testing: selection of reference values and interpretative strategies. Am Rev Respir Dis 1991; 144: 1202-1218.

11. Stocks J, Quanjer PH. Reference values for residual volume, functional residual capacity and total lung capacity ATS Workshop on Lung Volume Measurements. Official statement of the European Respiratory Society. Eur Respir J 1995; 8: 492-506.

12. Quanjer PH, Sly PD, Stocks J. Respiratory function measurements in infants: symbols, abbreviations and units. Eur Respir J 1995; 8: 1039-1056.

13. Quanjer PH, Sly PD, Stocks J. Uniform symbols, abbreviations, and units in paediatric pulmonary function testing. Paediatr Pulmonol 1997; 24: 2-11. 